Article

\title{
Impact of the Method Used to Select Gas Exchange Data for Estimating the Resting Metabolic Rate, as Supplied by Breath-by-Breath Metabolic Carts
}

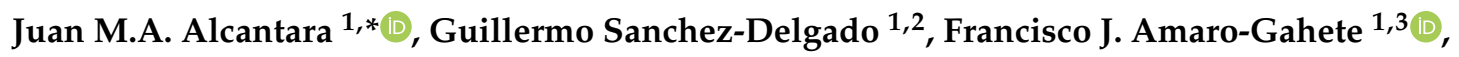 \\ Jose E. Galgani ${ }^{4}$ and Jonatan R. Ruiz ${ }^{1}$ (D) \\ 1 PROFITH “PROmoting FITness and Health Through Physical Activity” Research Group, Sport and Health \\ University Research Institute (iMUDS), Department of Physical and Sports Education, \\ Faculty of Sport Sciences, University of Granada, 18011 Granada, Spain; gsanchezdelgado@ugr.es (G.S.-D.); \\ amarof@ugr.es (F.J.A.-G.); ruizj@ugr.es (J.R.R.) \\ 2 Pennington Biomedical Research Center, Baton Rouge, LA 70808, USA \\ 3 EFFECTS-262 Research Group, Department of Physiology, School of Medicine, University of Granada, \\ 18011 Granada, Spain \\ 4 Departamento de Ciencias de la Salud, Carrera de Nutrición y Dietética, Facultad de Medicina, \\ Pontificia Universidad Católica de Chile, Santiago, Chile; Departamento de Nutrición, Diabetes y \\ Metabolismo, Facultad de Medicina, Universidad Pontificia Católica de Chile, 8330023 Santiago, Chile.; \\ jgalgani@uc.cl \\ * Correspondence: alcantarajma@ugr.es; Tel.: +34-958-244-353
}

Received: 23 December 2019; Accepted: 10 February 2020; Published: 14 February 2020

\begin{abstract}
The method used to select representative gas exchange data from large datasets influences the resting metabolic rate (RMR) returned. This study determines which of three methods yields the lowest RMR (as recommended for use in human energy balance studies), and in which method the greatest variance in RMR is explained by classical determinants of this variable. A total of 107 young and 74 middle-aged adults underwent a $30 \mathrm{~min}$ RMR examination using a breath-by-breath metabolic cart. Three gas exchange data selection methods were used: (i) steady state (SSt) for 3, 4, 5, or $10 \mathrm{~min}$, (ii) a pre-defined time interval (TI), i.e., 6-10, 11-15, 16-20, 21-25, 26-30, 6-25, or 6-30 min, and (iii) "filtering", setting thresholds depending on the mean RMR value obtained. In both cohorts, the RMRs yielded by the SSt and filtering methods were significantly lower $(p<0.021)$ than those yielded by the TI method. No differences in RMR were seen under the different conditions of the SSt method, or of the filtering method. No differences were seen between the methods in terms of the variance in RMR explained by its classical determinants. In conclusion, the SSt and filtering methods return the lowest RMRs and intra-measurement coefficients of variation when using breath-by-breath metabolic carts.
\end{abstract}

Keywords: resting energy expenditure; CCM express; CPX Ultima CardiO2; indirect calorimetry; macronutrient oxidation

\section{Introduction}

The resting metabolic rate (RMR) is the lowest energy expenditure of a person who is awake [1], after at least $12 \mathrm{~h}$ of fasting, being in physical rest, and in a state of mental relaxation in an ambient environmental temperature; it accounts for some $60-70 \%$ of the total daily energy expenditure [2]. The assessment of RMR is important when studying human energy balance, both in clinical and research settings [2,3]. Indirect calorimetry is the reference method for assessing RMR [2,4-6], which is estimated from the consumption of oxygen $\left(\mathrm{VO}_{2}\right)$ and the production of carbon dioxide $\left(\mathrm{VCO}_{2}\right)[6]$. The measurement of $\mathrm{VO}_{2}$ and $\mathrm{VCO}_{2}$, together with urinary nitrogen excretion, also allows for the 
estimation of the nutrient (carbohydrate and fat) oxidation rate [7]. Indeed, the $\mathrm{VCO}_{2} / \mathrm{VO}_{2}$ ratio, i.e., the respiratory quotient (RQ), is an indicator of the relative predominance of fat (FATOx) and carbohydrate (CHOOx) oxidation.

The assessment of RMR using indirect calorimetry is normally performed over a 10-30 min period. It is widely assumed that the first $5 \mathrm{~min}$ of data recorded should be discarded [8,9]. A short (e.g., $5 \mathrm{~min}$ ) steady respiratory state period, i.e., a period in which the indirect calorimetry record is markedly stable, then has to be selected from the remaining dataset for estimating the RMR $[6,7,10]$. The assumption that steady state (SSt) methods for gas exchange data selection provide a better estimate of RMR than the other methods available arose from studies performed in ventilated patients [10]. However, there is no strong evidence that the same can be assumed in healthy, non-ventilated subjects-and indeed different methods have been used. These methods can be grouped into three categories: (i) the selection of an SSt (defined as that providing a coefficient of variance [CV] of $<10 \%$ for $\mathrm{VO}_{2}, \mathrm{VCO}_{2}$, and minute ventilation [VE], and of $<5 \%$ for RQ [11]), (ii) the selection of a pre-defined time interval (TI), without taking the stability of the results obtained into consideration [11], and (iii) "filtering", in which data above or below a given RMR threshold are discarded. Both the SSt and TI methods can be used under different time conditions [1]. Unfortunately, the use of different methods for gas exchange data selection could result in different estimates of RMR and nutrient oxidation rates being made [1,11,12]. For instance, in a study involving healthy subjects, Irving et al. [1] reported RMR estimates made by the SSt and TI methods to differ by some -101 to $+121 \mathrm{kcal} /$ day.

Certainly, SSt-based RMR estimates are usually lower than those provided by the TI method [1,11]. Given the above definition of RMR [1], it has been proposed that the lowest estimates obtained by the SSt method should be deemed more accurate than those provided by TI. However, the under-estimation of the homeostatic RMR cannot be ruled out in the SSt method, nor has any study checked whether the filtering methods for data selection provide lower RMR estimates than either SSt or TI.

The present work examines whether the SSt, TI, or filtering method yields the lowest RMR value in healthy, non-ventilated subjects, and determines in which method the greatest variance in RMR is explained by the classical determinants of this variable (i.e., body weight, body composition, and sex) [13].

\section{Materials and Methods}

\subsection{Study Subjects}

The participants of this retrospective study were 107 young adults (72 women) enrolled in the ACTIBATE study [14] and 74 middle-aged adults (39 women) enrolled in the FIT-AGEING study [15]. Detailed information about the methodology of the aforementioned studies can be found elsewhere [14,15]. Briefly, the inclusion criteria were: (i) being physically inactive $(<20 \mathrm{~min}$ of moderate-vigorous physical activity on $<3$ days/week), (ii) having a stable body weight (change $<3 \mathrm{~kg}$ over the last 3 months [ACTIBATE] or $<5 \mathrm{~kg}$ over the last 5 months [FIT-AGEING]), (iii) not being enrolled in a weight loss program, (iv) not being a smoker, (v) not suffering from an acute or chronic illness, and (vi) not being pregnant. The ACTIBATE study protocol was approved by the Committee for Research Involving Human Subjects at the University of Granada (Reference \#924) and the Servicio Andaluz de Salud (Centro de Granada, CEI-Granada), while the FIT-AGEING was approved by the Human Research Ethics Committee of the Junta de Andalucia (0838-N-2017). Both studies were performed in accordance with the Declaration of Helsinki (2013 revision) and registered on the clinicaltrials.gov platform (IDs: NCT02365129 for the ACTIBATE study and NCT03334357 for the FIT-AGEING study). Oral and written informed consent was obtained from all the subjects before their enrolment.

\subsection{Procedures}

In both the above studies, subjects underwent a $30 \mathrm{~min}$ indirect calorimetry assessment of RMR at rest, early in the morning, following an overnight fast. All subjects were instructed to refrain from 
moderate $(24 \mathrm{~h})$ and vigorous physical activity $(48 \mathrm{~h})$ before the test day. On the previous evening, all subjects consumed a standardized meal of an egg omelet, boiled rice, and tomato sauce (ad libitum amounts). They were also instructed to avoid physical activity after they woke up, and to come to the research center by car or bus early in the morning, having had no breakfast (ensuring a $\sim 12 \mathrm{~h}$ fast). Upon arrival, and after confirming their compliance with these above conditions, body weight and height were measured using a Seca model 799 electronic column scale (seca GmbH \& Co. KG, Hamburg, Germany) with subjects barefoot and wearing light clothing. Thereafter, the subjects laid on a bed in the supine position for 20-30 $\mathrm{min}$. Gas exchange data were then recorded by indirect calorimetry for $30 \mathrm{~min}$ in a quiet room with dim lighting, controlled at $22-24{ }^{\circ} \mathrm{C}$ and $35-45 \%$ relative humidity [8]. During this time the subjects were covered with a bed sheet and instructed to remain silent, stay awake, avoid fidgeting, and to breathe normally.

\subsection{Gas Exchange Assessments}

Gas exchange was recorded using either a CCM Express or a CPX Ultima CardiO2 (two different devices were used only in the middle-aged adults cohort) breath-by-breath metabolic cart (Medical Graphics Corp, St. Paul, MN, USA). Both instruments require the use of a face mask equipped with a Directconnect ${ }^{\mathrm{TM}}$ flow sensor (Medical Graphics Corp, St. Paul, MN, USA). Both determine $\mathrm{VCO}_{2}$ using a non-dispersive infrared analyzer, and both determine $\mathrm{VO}_{2}$ using a galvanic fuel cell [16]. The flow rate was calibrated using a $3 \mathrm{~L}$ syringe at the beginning of every test. The gas analyzers were calibrated before each measurement using standard gases according to the manufacturers' instructions [16].

\subsection{Methods for Gas Exchange Data Selection}

The collected gas exchange data were processed using MGCDiagnostic ${ }^{\circledR}$ Breeze Suite 8.1.0.54 SP7 software (Medical Graphics Corp., St. Paul, MN, USA) to yield a data point for each variable for every minute (i.e., the means of all ventilation data (per minute ventilation data $-p M V D$ ) for each particular minute). The first $5 \mathrm{~min}$ of data were discarded [8,9]; the remaining $25 \mathrm{~min}$ period dataset was processed using three different methods to select representative gas exchange data for determining the RMR and nutrient oxidation rate.

\subsubsection{Time Interval Method}

Short TIs of 6-10 min, 11-15 min, 16-20 min, 21-25 min, and 26-30 min, and long TIs of 6-25 min and 6-30 min were established [11], and the means of the $p$ MVD values for all variables available for these time periods calculated. These processed data were used to calculate the RMR and nutrient oxidation rate (see below for details).

\subsubsection{Steady-State Time Method}

The CVs of $\mathrm{VO}_{2}, \mathrm{VCO}_{2}, \mathrm{VE}$, and $\mathrm{RQ}$ were calculated for every period of 3, 4, 5, and $10 \mathrm{~min}$ (e.g., for the $3 \mathrm{~min}$ SSt we processed all the $25 \mathrm{~min}$ period datasets and we examined the 6th to 8 th $\mathrm{min}$ period, the 7th to 9 th period, etc.) and the mean $\mathrm{CVs}$ for each variable calculated for each time period. The periods selected for the final analyses were those with the lowest mean CV for each (e.g., from the 3 min SSt examined periods, we selected the 7th to 9th) [11]. The means of the available $p$ MVD values for these time periods were then calculated. These processed data were used to calculate the RMR and nutrient oxidation rate (see below for details).

\subsubsection{Filtering Method}

The $p \mathrm{MVD}$ values for $\mathrm{VO}_{2}$ and $\mathrm{VCO}_{2}$ for the entire $25 \mathrm{~min}$ data collection period-i.e., with no division into SSt or TI periods-were used to calculate the mean 25 min RMR (see below for details). Furthermore, $p$ MVD RMR values were also calculated, discarding either (i) those values $<85 \%$ or $>115 \%$ of the mean $_{25}$ min RMR (low filter), (ii) $<90 \%$ or $>110 \%$ of the mean 25 min RMR (medium filter), 
or (iii) $<95 \%$ or $>105 \%$ of the mean 25 min $R M R$ (strong filter). For the minutes that passed these filters, the means were calculated for all $p$ MVD values available.

\subsection{Calculating the Resting Metabolic and Nutrient Oxidation Rates}

Weir's equation (assuming zero urinary nitrogen excretion) [17] was used to calculate RMR values from the mean $p \mathrm{MVD}$ for $\mathrm{VO}_{2}$ and $\mathrm{VCO}_{2}$ obtained with each gas exchange data selection method. The FATOx and CHOOx rates were calculated using Frayn's stoichiometric equations [18], also assuming zero urinary nitrogen excretion. Finally, the mean RMR, RQ, $\mathrm{VO}_{2}, \mathrm{VCO}_{2}, \mathrm{FATOx}$, and $\mathrm{CHOOx}$ were calculated for all gas exchange data selection methods under each different condition.

\subsection{Body Composition}

Body composition was determined by dual energy X-ray absorptiometry using a Discovery Wi device (Hologic, Inc., Bedford, MA, USA). Quality controls, the positioning of participants and analysis of the results were performed according to the manufacturer's recommendations.

\subsection{Statistical analysis}

Results are presented as mean \pm SD unless otherwise stated. All analyses were conducted using the Statistical Package for the Social Sciences v.22.0 (IBM SPSS Statistics, IBM Corporation, Chicago, IL, USA). Significance was set at $p<0.05$. Repeated-measures analysis of variance (ANOVA) with a post-hoc Bonferroni test was used to detect differences in $\mathrm{RMR}, \mathrm{RQ}, \mathrm{VO}_{2}, \mathrm{VCO}_{2}, \mathrm{FATOx}$, and CHOOx estimates across the methods for gas exchange data selection. The $\mathrm{CVs}$ for $\mathrm{VO}_{2}, \mathrm{VCO}_{2}, \mathrm{RQ}$, and $\mathrm{VE}$ obtained via the different methods were also compared. Two different ANOVA models were used: one with four levels of fixed factors (i.e., short TI, long TI, SSt, and filtering), and one with 14 levels (all methods and their different conditions).

The differences between the methods in terms of the variance in RMR explained by its classical determinants (i.e., body weight, body composition [lean and fat masses], and sex) [13] were examined by either simple linear regression (associations between RMR and body weight) or multiple linear regression (associations between RMR and sex and body weight; RMR and sex and lean and fat masses).

\section{Results}

Table 1 provides descriptive data for the subjects in both cohorts.

Table 1. Subject descriptive characteristics.

\begin{tabular}{|c|c|c|c|c|c|c|c|c|}
\hline & \multicolumn{4}{|c|}{ Young Adults $(n=107)$} & \multicolumn{4}{|c|}{ Middle-Aged Adults $(n=74)$} \\
\hline & & Min & Max & $\begin{array}{c}\text { Percentile } \\
10-90\end{array}$ & & Min & Max & $\begin{array}{c}\text { Percentile } \\
10-90\end{array}$ \\
\hline Age (years) & $22.2 \pm 2.2$ & 18.2 & 26.6 & $19.1-25.2$ & $53.5 \pm 5.3$ & 45.0 & 66.0 & $47.0-61.7$ \\
\hline \multicolumn{9}{|l|}{$\operatorname{Sex}(n \%)$} \\
\hline Women & 72,67 & & & & 39,53 & & & \\
\hline Men & 35,33 & & & & 35,47 & & & \\
\hline \multicolumn{9}{|l|}{ Metabolic cart used ( $n \%)$} \\
\hline CCM Express & 46,43 & & & & 0,0 & & & \\
\hline CPX Ultima CardiO2 & 61,57 & & & & 74,100 & & & \\
\hline Body weight (kg) & $69.3 \pm 15.9$ & 45.0 & 118.5 & $52.2-90.6$ & $75.7 \pm 15.0$ & 50.6 & 110.7 & $57.8-94.6$ \\
\hline Height $(\mathrm{cm})$ & $167.8 \pm 8.7$ & 148.5 & 195.1 & $157.2-180$ & $167.8 \pm 9.8$ & 148.3 & 189.8 & $155.8-181.6$ \\
\hline BMI $\left(\mathrm{kg} / \mathrm{m}^{2}\right)$ & $24.5 \pm 4.4$ & 17.2 & 38.4 & $19.4-30.9$ & $26.7 \pm 3.8$ & 19.0 & 38.0 & $22.0-31.7$ \\
\hline Waist circumference $(\mathrm{cm})$ & $79.8 \pm 13.2$ & 58.0 & 125.6 & $65.0-97.8$ & $95.1 \pm 11.7$ & 68.6 & 118.7 & $79.2-107.8$ \\
\hline Lean mass $(\mathrm{kg})$ & $41.4 \pm 9.5$ & 28.1 & 66.8 & $31.0-55.2$ & $43.5 \pm 11.7$ & 22.7 & 63.6 & $30.5-59.6$ \\
\hline Fat mass $(\mathrm{kg})$ & $24.0 \pm 8.9$ & 9.9 & 51.7 & $14.6-36.4$ & $30.0 \pm 8.4$ & 14.5 & 55.8 & $20.6-40.7$ \\
\hline Fat mass $(\%)$ & $35.0 \pm 7.8$ & 15.3 & 51.9 & $26.2-44.4$ & $39.9 \pm 9.1$ & 23.0 & 59.4 & $26.7-51.1$ \\
\hline
\end{tabular}




\subsection{Influence of the Gas Exchange Data Selection Method on Estimates of RMR, RQ and Nutrient Oxidation}

Figure 1 shows the RMR and RQ estimates yielded by the gas exchange data selection methods for both the young and middle-aged adults. In the young adults, the short and long TI methods provided higher mean RMR estimates than either the SSt or filtering methods (taking all conditions together; post-hoc Bonferroni $p<0.001$; Figure 1A). In the middle-aged adults they also provided higher mean RMR estimates than the filtering method (taking all conditions together; post-hoc Bonferroni $p<0.001$; Figure 1B). No differences were seen between the RMR estimates yielded by the short and long TIs, nor between the SSt and the filtering methods (taking all conditions together) in either the young or the middle-aged adults (all post-hoc Bonferroni $p=1.000$; Figure 1A,B). For the young adults, the lowest mean RMR values were obtained with the SSt 4 min method (1440 kcal/day; Figure 1E); however, the SSt 4 min method was only statistically different from the TI 6-10 min and the TI 11-15 min. In the middle-aged adults the lowest mean RMR values were provided by the strong-filter method (1493 kcal/day; Figure 1F); the strong-filter method was statistically different from all the different methods, with the exception of the TI 11-15 min, and the SSt 3, 4, and 5 min conditions. Table S1 shows the comparisons (i.e., post-hoc Bonferroni) between the different methods. Similar patterns were observed when analyzing the influence of gas exchange data selection method on $\mathrm{VO}_{2}$ and $\mathrm{VCO}_{2}$ estimates (Figure S1).

Lastly, the periods in which the SSt were achieved (with the different SSt methods applied) in the young and the middle-aged adults are presented in Figure S2. We observed that $~ 50 \%$ of young and middle-aged adults achieved their SSts (i.e., the one presenting lower mean CV) during the first half of the $30 \mathrm{~min}$ indirect calorimetry assessment. On the other hand, we found differences between the first steady state achieved (i.e., the first period in which the $\mathrm{CVs}$ of $\mathrm{VO}_{2}<10, \mathrm{VCO}_{2}<10, \mathrm{VE}<10$, and $\mathrm{RQ}<5$ ) and the "best" SSt achieved (i.e., the period with the lowest mean CVs) in RMR estimation in young adults (Figure S3).

The RQ estimates yielded by the short TI method were significantly higher than all others in the young adult cohort (all post-hoc Bonferroni $p<0.002$; Figure 1C) and that filtering method (taking all conditions together) in the middle-aged adults cohort (post-hoc Bonferroni $p=0.038$; Figure 1D). Moreover, the long TI method provided higher RQ estimates than the SSt and filtering methods (taking all conditions together) in the young adult (both Bonferroni post-hoc $p<0.013$; Figure 1C). No differences in RQ estimates were seen when comparing the SSt and filtering methods (taking all conditions together) in either the young or the middle-aged adults (post-hoc Bonferroni $p=1.000$; Figure 1C,D). Furthermore, the long TI and the SSt were not significantly different than either the short TI method or the filtering methods (taking all conditions together) in the middle-aged adults (Figure 1D). The lowest mean RQ values were obtained when using the strong-filter method (0.84) in the young adults (Figure $1 G$ ). However, the strong-filter method was only statistically different from the TI 26-30 min and the TI 6-30 min. The lowest mean RQ values were obtained when using the SSt 3 min method (0.80) in the middle-aged adults (Figure $1 \mathrm{H}$ ). However, no statistical differences were observed. As expected, the data selection methods yielding higher RQ estimates also provided higher CHOOx and lower FATOx estimates, and vice versa (Figure 2). 
Young adults $(n=107)$

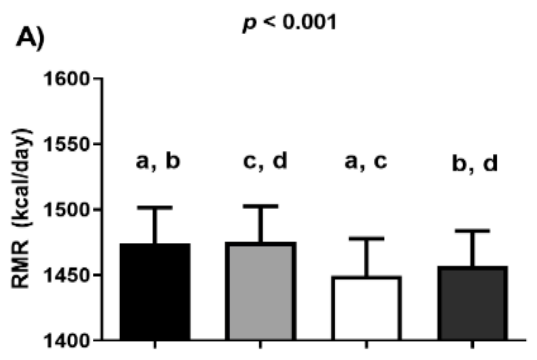

C)

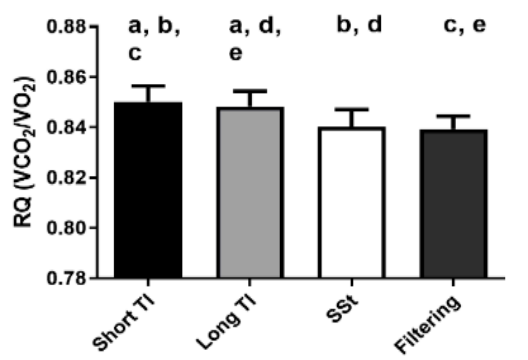

E)

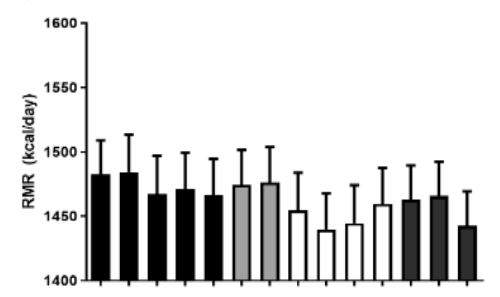

G)

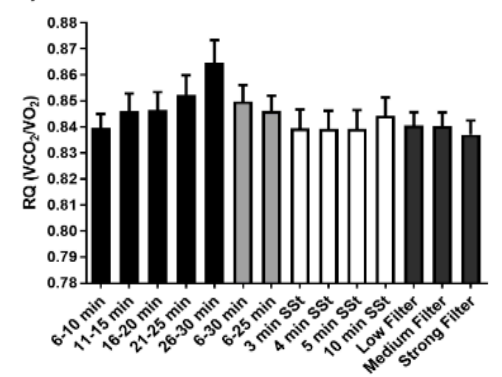

Middle-aged adults $(n=74)$

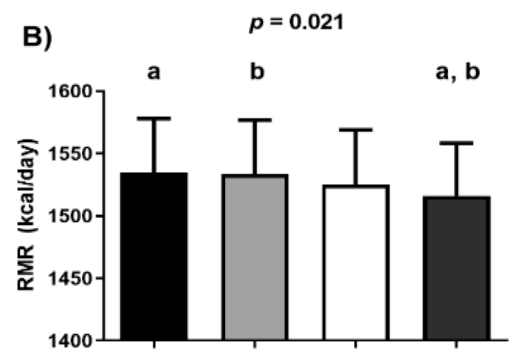

D)

$p=0.024$

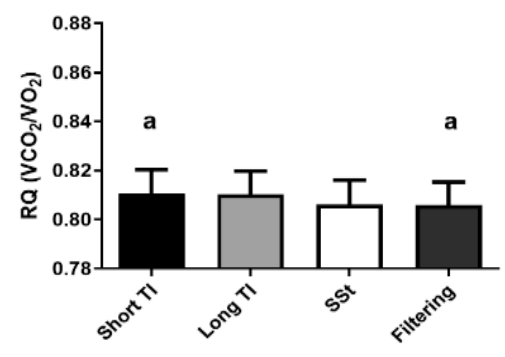

F)

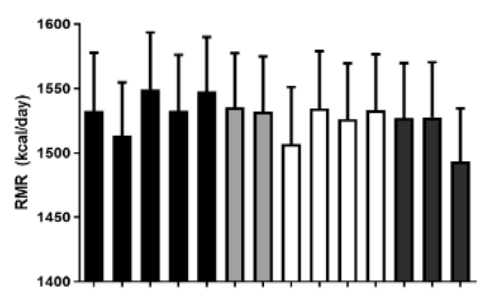

H)

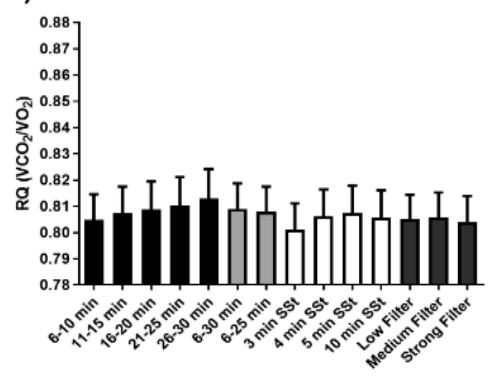

Figure 1. Differences among gas exchange data selection methods with respect to resting metabolic rate (RMR) and respiratory quotient (RQ) estimates. Black columns represent short time interval (TI) periods (i.e., the means of the per minute ventilation data ( $p$ MVD]) values for all variables available for these time periods, panels A-D; the $p$ MVD values for each short TI period, panels E,F). Light grey columns represent long TI periods (i.e., the means of the $p$ MVD values for all variables available for these time periods, panels A-D; the means of the $p$ MVD values for each long TI period, panels E,F). White columns represent steady state (SSt) periods (i.e., the means of the $p$ MVD values for all variables available for these SSt periods, panels A-D; the means of the $p$ MVD values for each SSt period, panels E,F). Dark grey columns represent filtering methods (i.e., the means of the $p$ MVD values for all variables available for these filtering periods, panels A-D; the means of the $p$ MVD values for each filtering period, panels $\mathbf{E}, \mathbf{F}$ ). $p$-values come from repeated-measures analysis of variance (ANOVA). Identical indicatory letters highlight differences as determined by post-hoc Bonferroni analysis. Data are presented as mean and standard error of the mean (SEM). Min: minutes; $\mathrm{VCO}_{2}$ : production of carbon dioxide; $\mathrm{VO}_{2}$ : consumption of oxygen. 
Young adults $(n=107)$

A)

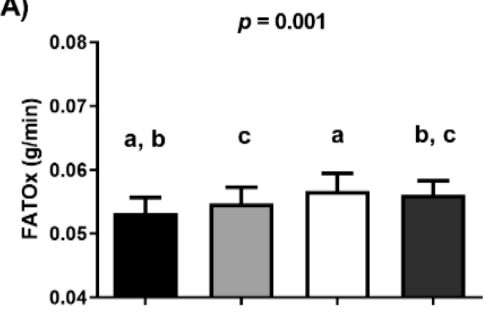

$p<0.001$

C)

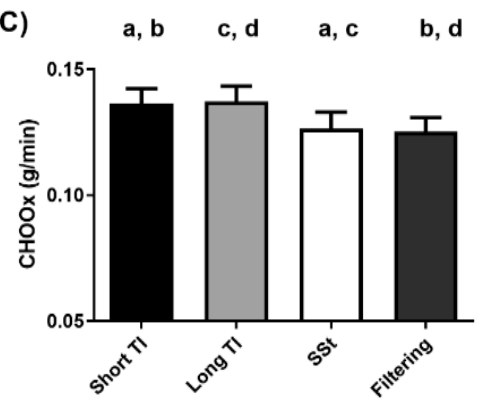

E)

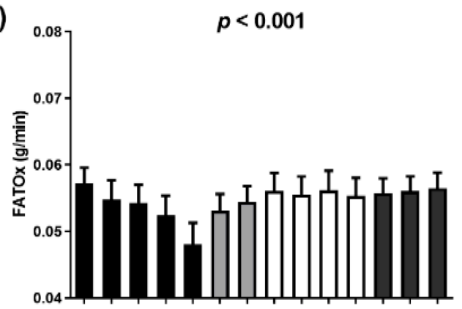

G)

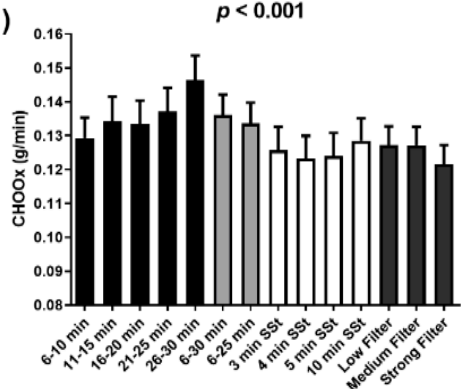

Middle-aged adults $(n=74)$

B)

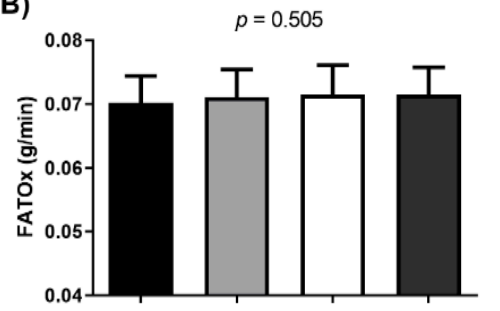

$p=0.010$

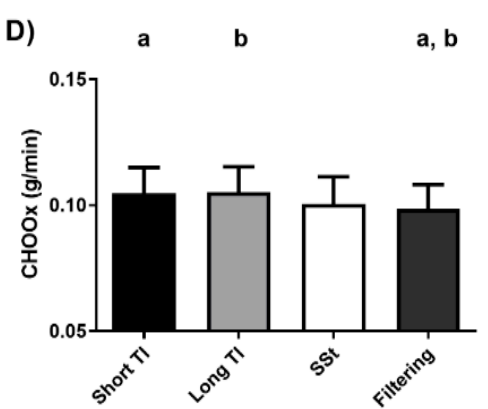

F)

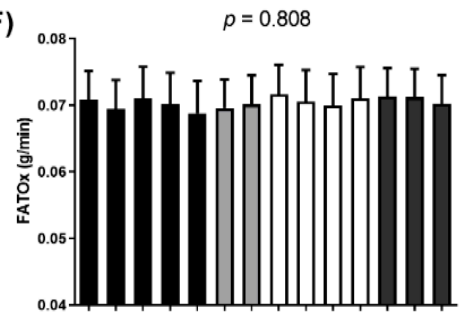

H)

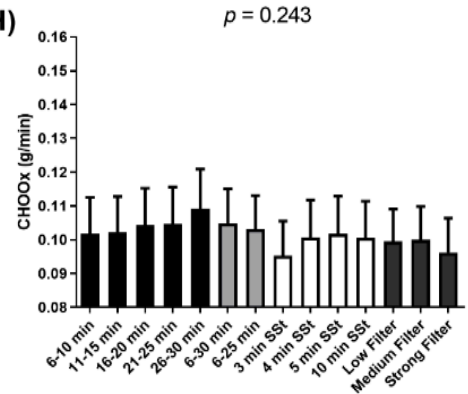

Figure 2. Differences among gas exchange data selection methods with respect to fat oxidation (FATOx) and carbohydrate oxidation (CHOOx) rates. Black columns represent short time interval (TI) periods (i.e., the means of the per minute ventilation data $[p \mathrm{MVD}]$ values for all variables available for these time periods, panels A-D; the $p$ MVD values for each short TI period, panels E,F). Light grey columns represent long TI periods (i.e., the means of the $p \mathrm{MVD}$ values for all variables available for these time periods, panels A-D; the means of the $p$ MVD values for each long TI period, panels E,F). White columns represent steady state (SSt) periods (i.e., the means of the $p$ MVD values for all variables available for these SSt periods, panels A-D; the means of the $p$ MVD values for each SSt period, panels E,F). Dark grey columns represent filtering methods (i.e., the means of the $p$ MVD values for all variables available for these filtering periods, panels A-D; the means of the $p$ MVD values for each filtering period, panels E,F). $p$-values come from repeated-measures analysis of variance (ANOVA). Identical indicatory letters highlight differences as determined by post-hoc Bonferroni analysis. Data are presented as mean and standard error of the mean (SEM). Min: minutes. 
3.2. Differences between the Methods in Terms of the Variance in RMR Explained by Its Classical Determinants

The variance in RMR explained by body weight (taking all conditions together) was 36\%, 36\%, $34 \%$ and $38 \%$ for the short TI, long TI, SSt, and filtering methods respectively in young adults, and $50 \%, 51 \%, 52 \%$ and $51 \%$ respectively in the middle-aged adults. The most explained variance was obtained using the low-filter method (40\%) in young adults and the TI 6-10 min method (54\%) in the middle-aged adults.

The variance explained increased to $34-45 \%$ and $54-68 \%$ in the young and middle-aged adults respectively after including subject sex in the model (Table 2). The most explained variance was obtained with the low-filter method in young adults, and both the TI 21-25 min and the medium-filter methods in the middle-aged adults. However, little difference was seen among the methods in terms of the variance explained by the classical determinants of RMR (Table 2).

A further model including subject sex, lean, and fat masses increased the variance in RMR explained by $\sim 5 \%$ in the young adults, but not in the middle-aged adults (Table 3 ). The low-filter method explained the greatest variance in RMR in the young adults, and the TI 21-25 min method did so in the middle-aged adults. However, once again, little difference was seen among the methods in terms of the variance explained by the classical determinants of RMR (Table 3). 
Table 2. Variance in resting metabolic rate (RMR) explained by sex and body weight in each of the gas exchange data selection methods

\begin{tabular}{|c|c|c|c|c|c|c|c|c|c|c|c|c|}
\hline \multirow{3}{*}{ Method of Data Selection } & \multicolumn{6}{|c|}{ Young Adults $(n=107)$} & \multicolumn{6}{|c|}{ Middle-Aged Adults $(n=74)$} \\
\hline & \multirow[b]{2}{*}{ Model $\mathbf{R}^{2}$} & \multirow[b]{2}{*}{ Constant } & \multicolumn{2}{|c|}{ Sex } & \multicolumn{2}{|c|}{ Weight (kg) } & \multirow[b]{2}{*}{ Model $\mathbf{R}^{2}$} & \multirow[b]{2}{*}{ Constant } & \multicolumn{2}{|c|}{ Sex } & \multicolumn{2}{|c|}{ Weight (kg) } \\
\hline & & & $\beta$ & $p$ & $\beta$ & $p$ & & & $\beta$ & $p$ & $\beta$ & $p$ \\
\hline TI 6-10 min & 0.44 & 1232 & -171.6 & 0.001 & 7.8 & $<0.001$ & 0.67 & 1503 & -424.0 & 0.001 & 8.9 & 0.001 \\
\hline TI $11-15 \mathrm{~min}$ & 0.40 & 1340 & -214.4 & $<0.001$ & 7.3 & $<0.001$ & 0.67 & 1616 & -418.5 & 0.001 & 7.1 & 0.004 \\
\hline TI 16-20 min & 0.40 & 1300 & -214.5 & $<0.001$ & 7.6 & $<0.001$ & 0.60 & 1758 & -445.2 & 0.001 & 6.2 & 0.031 \\
\hline TI $21-25 \mathrm{~min}$ & 0.36 & 1342 & -199.5 & 0.001 & 6.7 & $<0.001$ & 0.68 & 1935 & -507.5 & 0.001 & 4.9 & 0.051 \\
\hline TI 26-30 min & 0.34 & 1208 & -155.7 & 0.009 & 7.5 & $<0.001$ & 0.54 & 1754 & -409.8 & 0.001 & 5.5 & 0.058 \\
\hline TI 6-30 min & 0.42 & 1284 & -191.2 & 0.001 & 7.4 & $<0.001$ & 0.66 & 1713 & -441.0 & 0.001 & 6.5 & 0.010 \\
\hline TI 6-25 min & 0.43 & 1303 & -200.0 & $<0.001$ & 7.3 & $<0.001$ & 0.67 & 1703 & -448.8 & 0.001 & 6.8 & 0.008 \\
\hline SSt $3 \mathrm{~min}$ & 0.39 & 1245 & -194.9 & 0.001 & 7.8 & $<0.001$ & 0.66 & 1669 & -456.7 & 0.001 & 7.1 & 0.008 \\
\hline SSt 4 min & 0.39 & 1169 & -167.5 & 0.004 & 7.9 & $<0.001$ & 0.67 & 1786 & -481.1 & 0.001 & 6.4 & 0.016 \\
\hline SSt $5 \mathrm{~min}$ & 0.36 & 1301 & -207.0 & 0.001 & 7.1 & $<0.001$ & 0.66 & 1763 & -464.1 & 0.001 & 6.2 & 0.016 \\
\hline SSt $10 \mathrm{~min}$ & 0.41 & 1232 & -182.8 & 0.001 & 7.7 & $<0.001$ & 0.66 & 1681 & -443.8 & 0.001 & 7.0 & 0.008 \\
\hline Low-filter & 0.45 & 1188 & -170.6 & 0.001 & 8.1 & $<0.001$ & 0.67 & 1701 & -442.9 & 0.001 & 6.6 & 0.009 \\
\hline Medium-filter & 0.43 & 1209 & -170.8 & 0.001 & 7.8 & $<0.001$ & 0.68 & 1725 & -447.5 & 0.001 & 6.4 & 0.010 \\
\hline Strong-filter & 0.44 & 1218 & -182.0 & 0.001 & 7.7 & $<0.001$ & 0.67 & 1684 & -431.7 & 0.001 & 6.2 & 0.012 \\
\hline
\end{tabular}

Unstandardized beta and $p$-values (significant values in bold) from multiple regression analyses, in which sex and body weight were included as independent variables, and the RMR estimates yielded by the different methods of gas exchange data selection were included as dependent variables. Sex: $1=$ men; $2=$ women. 
Table 3. Variance in resting metabolic rate (RMR) explained by sex, lean mass (LM) and fat mass (FM) in each of the gas exchange data selection methods.

\begin{tabular}{|c|c|c|c|c|c|c|c|c|c|c|c|c|c|c|c|c|}
\hline \multirow{3}{*}{ Method of Data Selection } & \multicolumn{8}{|c|}{ Young Adults $(n=107)$} & \multicolumn{8}{|c|}{ Middle-Aged Adults $(n=74)$} \\
\hline & \multirow[b]{2}{*}{ Model $\mathbf{R}^{2}$} & \multirow[b]{2}{*}{ Constant } & \multicolumn{2}{|c|}{ Sex } & \multicolumn{2}{|c|}{ LM (kg) } & \multicolumn{2}{|c|}{ FM (kg) } & \multirow[b]{2}{*}{ Model $\mathbf{R}^{2}$} & \multirow[b]{2}{*}{ Constant } & \multicolumn{2}{|c|}{ Sex } & \multicolumn{2}{|c|}{ LM (kg) } & \multicolumn{2}{|c|}{ FM (kg) } \\
\hline & & & $\beta$ & $p$ & $\beta$ & $p$ & $\beta$ & $p$ & & & $\beta$ & $p$ & $\beta$ & $p$ & $\beta$ & $p$ \\
\hline TI 6-10 min & 0.47 & 668 & -3.5 & 0.967 & 18.7 & $<0.001$ & 2.1 & 0.450 & 0.66 & 1436 & -400.4 & 0.001 & 10.5 & 0.020 & 8.4 & 0.011 \\
\hline TI $11-15 \mathrm{~min}$ & 0.45 & 483 & 39.2 & 0.683 & 23.4 & $<0.001$ & -1.2 & 0.697 & 0.67 & 1583 & -407.0 & 0.001 & 8.0 & 0.050 & 6.8 & 0.022 \\
\hline TI $16-20 \mathrm{~min}$ & 0.44 & 497 & 23.2 & 0.814 & 22.8 & $<0.001$ & -0.3 & 0.925 & 0.60 & 1533 & -370.0 & 0.001 & 10.4 & 0.029 & 4.3 & 0.210 \\
\hline TI $21-25 \mathrm{~min}$ & 0.41 & 542 & 37.0 & 0.702 & 21.7 & $<0.001$ & -1.2 & 0.697 & 0.68 & 1713 & -433.1 & 0.001 & 9.0 & 0.030 & 3.0 & 0.320 \\
\hline TI 26-30 min & 0.38 & 433 & 73.8 & 0.451 & 22.1 & $<0.001$ & -0.2 & 0.957 & 0.55 & 1500 & -324.4 & 0.005 & 10.2 & 0.034 & 3.3 & 0.346 \\
\hline TI 6-30 min & 0.47 & 525 & 34.0 & 0.701 & 21.7 & $<0.001$ & -0.2 & 0.955 & 0.66 & 1553 & -381.0 & 0.001 & 9.6 & 0.022 & 5.2 & 0.089 \\
\hline TI 6-25 min & 0.47 & 548 & 24.0 & 0.787 & 21.6 & $<0.001$ & -0.2 & 0.956 & 0.67 & 1566 & -402.5 & 0.001 & 9.5 & 0.024 & 5.6 & 0.065 \\
\hline SSt $3 \mathrm{~min}$ & 0.44 & 417 & 49.5 & 0.610 & 23.3 & $<0.001$ & -0.3 & 0.919 & 0.66 & 1657 & -452.0 & 0.001 & 7.6 & 0.085 & 7.0 & 0.029 \\
\hline SSt 4 min & 0.45 & 306 & 87.7 & 0.344 & 24.2 & $<0.001$ & -0.5 & 0.868 & 0.67 & 1695 & -451.0 & 0.001 & 8.2 & 0.060 & 5.7 & 0.073 \\
\hline SSt $5 \mathrm{~min}$ & 0.42 & 379 & 65.7 & 0.510 & 24.3 & $<0.001$ & -2.0 & 0.530 & 0.66 & 1694 & -441.0 & 0.001 & 7.7 & 0.073 & 5.7 & 0.066 \\
\hline SSt $10 \mathrm{~min}$ & 0.45 & 464 & 44.8 & 0.628 & 22.2 & $<0.001$ & 0.1 & 0.965 & 0.66 & 1534 & -394.1 & 0.001 & 9.9 & 0.023 & 5.7 & 0.069 \\
\hline Low-filter & 0.50 & 451 & 47.8 & 0.571 & 22.1 & $<0.001$ & 0.8 & 0.770 & 0.67 & 1577 & -400.9 & 0.001 & 9.1 & 0.030 & 5.6 & 0.066 \\
\hline Medium-filter & 0.48 & 424 & 62.0 & 0.471 & 22.7 & $<0.001$ & 0.1 & 0.989 & 0.67 & 1597 & -404.1 & 0.001 & 8.9 & 0.029 & 5.3 & 0.073 \\
\hline Strong-filter & 0.49 & 416 & 55.4 & 0.516 & 22.8 & $<0.001$ & -0.3 & 0.918 & 0.67 & 1534 & -381.2 & 0.001 & 9.1 & 0.025 & 4.9 & 0.094 \\
\hline
\end{tabular}

Unstandardized beta and $p$-values (significant values in bold) from multiple regression analyses, in which sex, LM and FM were included as independent variables, and the RMR estimates yielded by the different methods of gas exchange data selection were included as dependent variables. Sex: $1=$ men; $2=$ women. 


\section{Discussion}

The present results show that when using breath-by-breath metabolic carts, RMR, and RQ estimates yielded by the SSt and filtering methods are lower than those yielded by the TI method, while no differences were seen between the SSt and filtering methods. The variance in the RMR explained by its classical determinants (i.e., weight, body composition, and sex) was similar in all methods. These findings largely concurred in the cohorts of young adults and middle-aged adults examined, which further reinforced the consistency of the results.

\subsection{Influence of the Gas Exchange Data Selection Method on RMR, RQ and Nutrient Oxidation}

Given that RMR is defined as the lowest energy expenditure of a person who is awake [1], the present results suggest that the SSt and filtering methods provide better RMR estimates. These results are in line with those reported in the literature $[1,12]$ and concur with those reported in our previous study [11], in which lower RMR and RQ estimates were yielded by the SSt method than the TI method. As expected, the SSt method showed less variability in the results returned than the TI method (Figures S4 and S5). This supports the notion that the assessment of stable state respiratory gas exchange variables provides the best results $[8,19]$.

The SSt method purportedly reflects the baseline physiological state [10], thus reflecting the homeostatic RMR and nutrient oxidation rates [20,21]. Reeves et al. [19] reported that SSt measurements for assessing RMR were more accurate when taking data over short periods (e.g., $30 \mathrm{~min}$ ). In line with this, McClave et al. [10] reported that the RMR obtained with the SSt 5 min method provides an accurate representation of the $24 \mathrm{~h}$ total energy expenditure in bedridden hospitalized patients owing to their low level of physical activity. However, in healthy individuals, the RMR does not represent the $24 \mathrm{~h}$ total energy expenditure [6]. McClave et al. [10] recommended that: (i) a steady respiratory state should be considered reached when changes in the $\mathrm{CV}$ of $\mathrm{VO}_{2}$ and $\mathrm{VCO}_{2}$ are $<10 \%$ over a period of 5 consecutive min, (ii) RMR assessment should end when a steady respiratory state is achieved, and (iii) when a steady respiratory state cannot be reached, a more prolonged test ( $\geq 60 \mathrm{~min}$ ) becomes necessary. Reeves et al. [19] showed that reducing the steady respiratory state time period for data collection from 5 to 4 min resulted in acceptable RMR values. It has been suggested that reducing it to 3 min might underestimate the RMR [19], but in our previous study [11] no differences were seen between the RMRs provided by the SSt 3, 4, or 5 min conditions. The present results agree with our previous findings in a smaller sample of young adults (17 vs. the present 107) and middle-aged adults [11]. In addition, the present results for both independent cohorts were similar (i.e., replicated). As we mentioned previously, the assessment of RMR using indirect calorimetry is normally performed over a $30 \mathrm{~min}$ period, and our results suggest that the SSt becomes more stable (i.e., less variable) as the RMR measurement progresses (Figure S3). On the other hand, we observed that after $\sim 15 \mathrm{~min}$ of measurement, $\sim 50 \%$ of the young and the middle-aged adults achieved the SSt (Figure S2). Thus, based on our results we would recommend that when RMR is assessed using indirect calorimetry the measurement should last at least $30 \mathrm{~min}$, to obtain the most stable results as possible reducing the variability (Figure S3). Moreover, it could be interesting to test whether the stability increases (or not) if the RMR measurement lasts more than $30 \mathrm{~min}$.

To our knowledge, no other study has compared the TI, SSt, and filtering methods, rendering further comparisons impossible. However, the present results suggest that those provided by the filtering method are similar to those provided by the SSt method. It might therefore be used as an alternative in subjects in whom a steady respiratory state is not achieved (although the present subjects all reached a steady respiratory state, which might bias this suggestion).

Mean FATOx was higher and CHOOx lower, when determined via the SSt and filtering methods compared to the TI method, with no differences seen among the different SSt periods or among the different filtering conditions. In contrast, differences were observed among the TI periods in terms of both the RQ and nutrient oxidation rates, with an increase in the RQ seen over each test period (more pronounced in the young adult cohort-Figure 2). This gradual increase in the RQ, which might 
influence the nutrient oxidation rates estimates, may be related to the metabolic cart rather than subject factors or the method of gas exchange data selection used, since this increase was also observed in a previous study involving different subjects but using the same metabolic carts [11].

\subsection{Differences between the Methods in Terms of the Variance in RMR Explained by Its Classical Determinants}

For the middle-aged adults, the variance in RMR explained by its classical determinants across the gas exchange data selection methods was in line with the results of previous studies (36-56\% explained by body weight [3,22-24]), although in the young adult cohort the variance explained was less than in the majority of the aforementioned studies. When comparing with previous studies in which body composition was included in the regression model, the variance in RMR explained across methods in the present study was also lower. In fact, Müller et al. [25] reported $72 \%$ of the variance in RMR to be explained by sex, lean mass, and fat mass. Korth et al. [22] reported 75\% of the variance to be explained by lean mass alone, while Mifflin et al. [23] reported a value of $64 \%$. Galgani and Castro-Sepulveda [3] reported 75\% of the variance in RMR to be explained by fat-free mass, fat mass, and age. In the present study, the variance explained for the middle-aged adults was in line with the aforementioned studies (55-68\% depending on the gas exchange data selection method used-Table 3). The differences across studies might be related, to a greater or lesser extent, to the accuracy of the metabolic cart [16]. In fact, in that study [16] we compared the inter-day reliability and the congruent validity of the CCM Express and the CPX Ultima CardiO2. Firstly, we observed that the CCM Express metabolic cart is more reliable than the CPX Ultima CardiO2 (i.e., less RMR inter-day differences: $158 \pm 154 \mathrm{kcal} /$ day vs. $219 \pm 185 \mathrm{kcal} /$ day for the CCM Express and the CPX Ultima CardiO2 respectively) [16]. Secondly, we observed that the RMR values obtained using the CPX Ultima CardiO2 were higher than the values obtained using the CCM Express metabolic cart (mean difference between metabolic carts of $65 \pm 161 \mathrm{kcal} /$ day on study day 1 and $94 \pm 161 \mathrm{kcal} /$ day on study day 2) [16]. Thus, as mentioned, the differences across studies could be related to the accuracy of the metabolic cart, and it may be that neither of the breath-by-breath carts used (i.e., the CCM Express and CPX Ultima CardiO2) is sufficiently accurate for measuring RMR if the ability to explain the variance in RMR is taken as an indirect indicator of accuracy. However, the variance explained was quite similar for all the gas exchange data selection methods used; it may not, therefore, be "method-dependent".

The present results should be considered with some caution. The assessment of RMR was performed using two different breath-by-breath metabolic carts indiscriminately, both of which were equipped with a face-mask. The use of other metabolic carts or other gas collection systems (e.g., canopy collection) might influence the RMR results obtained [26,27]. Further studies involving different metabolic carts and gas collection systems, as well as with different subject populations, are needed to confirm the results.

\section{Conclusions}

The present findings suggest that when using CCM Express and CPX Ultima CardiO2 breath-by-breath metabolic carts, both the SSt and filtering methods yield the lowest RMR and $\mathrm{RQ}$ estimates with the lowest amount of variability (i.e., lowest intra-measurement coefficients of variation). Moreover, the filtering method might be a valid alternative for use with subjects who do not achieve a steady respiratory state.

Supplementary Materials: The following data are available online at http://www.mdpi.com/2072-6643/12/2/487/s1: Figure S1: Differences among methods for gas exchange data selection with respect to $\mathrm{VO}_{2}$ and $\mathrm{VCO}_{2}$, Figure S2: Period from the $30 \mathrm{~min}$ RMR measurement in which the SSt is achieved, Figure S3: Differences among 5 min SSt method achieved at different time lengths gas exchange data selection with respect to $R M R, V_{2}, V C O_{2}, R Q$, and Mean All CVs, Figures S4 and S5: Differences among gas exchange data selection with respect to the $\mathrm{CV}_{2} \mathrm{VO}_{2}, \mathrm{CV}$ $\mathrm{VCO}_{2}, \mathrm{CV} \mathrm{RQ}, \mathrm{CV}$ VE, and the Mean All CVs, and Table S1: post-hoc Bonferroni analysis. 
Author Contributions: Conceptualization, J.M.A.A., G.S.-D., and J.R.R; Data curation, J.M.A.A.; Formal analysis, J.M.A.A., G.S.-D., and J.R.R.; Methodology, J.M.A.A., G.S.-D., and J.R.R.; Writing-original draft, J.M.A.A.; Writing-review and editing, J.M.A.A., G.S.-D., F.J.A.-G., J.E.G., and J.R.R. All authors have read and agreed to the published version of the manuscript.

Funding: This study was supported by the Spanish Ministry of Economy and Competitiveness via the Fondo de Investigación Sanitaria del Instituto de Salud Carlos III (PI13/01393), Retos de la Sociedad (DEP2016-79512-R) and European Regional Development Funds (ERDF), the Spanish Ministry of Education (FPU 15/04059 and FPU14/04172), the Fundación Iberoamericana de Nutrición (FINUT), the Redes Temáticas de Investigación Cooperativa RETIC (Red SAMID RD16/0022), the AstraZeneca HealthCare Foundation, the University of Granada Plan Propio de Investigación 2016 Excellence Actions: Unit of Excellence on Exercise and Health (UCEES) and Plan Propio de Investigación 2018 and 2019 Programa Contratos-Puente and Plan Propio de Investigación 2018 Programa Perfeccionamiento de Doctores, and the Junta de Andalucía, Consejería de Conocimiento, Investigación y Universidades (ERDF: ref. SOMM17/6107/UGR), and the Fundación Alfonso Martín Escudero.

Acknowledgments: This study was part of a Ph.D thesis conducted within the framework of the Biomedicine Doctoral Studies Program of the University of Granada, Spain.

Conflicts of Interest: The authors declare no conflict of interest.

\section{References}

1. Irving, C.J.; Eggett, D.L.; Fullmer, S. Comparing Steady State to Time Interval and Non-Steady State Measurements of Resting Metabolic Rate. Nutr. Clin. Pract. 2017, 32, 77-83. [CrossRef] [PubMed]

2. Lam, Y.Y.; Ravussin, E. Indirect calorimetry: An indispensable tool to understand and predict obesity. Eur. J. Clin. Nutr. 2017, 71, 318-322. [CrossRef] [PubMed]

3. Galgani, J.E.; Castro-Sepulveda, M.A. Influence of a Gas Exchange Correction Procedure on Resting Metabolic Rate and Respiratory Quotient in Humans. Obesity 2017, 25, 1941-1947. [CrossRef] [PubMed]

4. Black, C.; Grocott, M.P.W.; Singer, M. Metabolic monitoring in the intensive care unit: A comparison of the Medgraphics Ultima, Deltatrac II, and Douglas bag collection methods. Br. J. Anaesth. 2015, 114, 261-268. [CrossRef] [PubMed]

5. Cooper, J.A.; Watras, A.C.; O’Brien, M.J.; Luke, A.; Dobratz, J.R.; Earthman, C.P.; Schoeller, D.A. Assessing validity and reliability of resting metabolic rate in six gas analysis systems. J. Am. Diet. Assoc. 2009, 109, 128-132. [CrossRef]

6. Da Rocha, E.E.M.; Alves, V.G.F.; da Fonseca, R.B.V. Indirect calorimetry: Methodology, instruments and clinical application. Curr. Opin. Clin. Nutr. Metab. Care 2006, 9, 247-256. [CrossRef]

7. Simonson, D.C.; DeFronzo, R.A. Indirect calorimetry: Methodological and interpretative problems. Am. J. Physiol. Metab. 2017, 258, E399-E412. [CrossRef]

8. Fullmer, S.; Benson-Davies, S.; Earthman, C.P.; Frankenfield, D.C.; Gradwell, E.; Lee, P.S.P.; Piemonte, T.; Trabulsi, J. Evidence analysis library review of best practices for performing indirect calorimetry in healthy and non-critically ill individuals. J. Acad. Nutr. Diet. 2015, 115, 1417-1446. [CrossRef]

9. Horner, N.K.; Lampe, J.W.; Patterson, R.E.; Neuhouser, M.L.; Beresford, S.A.; Prentice, R.L. Indirect Calorimetry Protocol Development for Measuring Resting Metabolic Rate as a Component of Total Energy Expenditure in Free-Living Postmenopausal Women. J. Nutr. 2001, 131, 2215-2218. [CrossRef]

10. McClave, S.A.; Spain, D.A.; Skolnick, J.L.; Lowen, C.C.; Kleber, M.J.; Wickerham, P.S.; Vogt, J.R.; Looney, S.W. Achievement of steady state optimizes results when performing indirect calorimetry. J. Parenter. Enter. Nutr. 2003, 27, 16-20. [CrossRef]

11. Sanchez-Delgado, G.; Alcantara, J.M.A.; Ortiz-Alvarez, L.; Xu, H.; Martinez-Tellez, B.; Labayen, I.; Ruiz, J.R. Reliability of resting metabolic rate measurements in young adults: Impact of methods for data analysis. Clin. Nutr. 2018, 37, 1618-1624. [CrossRef] [PubMed]

12. Borges, J.H.; Guerra-Júnior, G.; Gonçalves, E.M. Methods for data analysis of resting energy expenditure measured using indirect calorimetry. Nutrition 2019, 59, 44-49. [CrossRef] [PubMed]

13. Johnstone, A.M.; Murison, S.D.; Duncan, J.S.; Rance, K.A.; Speakman, J.R. Factors influencing variation in basal metabolic rate include fat-free mass, fat mass, age, and circulating thyroxine but not sex, circulating leptin, or triiodothyronine. Am. J. Clin. Nutr. 2005, 82, 941-948. [CrossRef] [PubMed] 
14. Sanchez-Delgado, G.; Martinez-Tellez, B.; Olza, J.; Aguilera, C.M.; Labayen, I.; Ortega, F.B.; Chillon, P.; Fernandez-Reguera, C.; Alcantara, J.M.A.; Martinez-Avila, W.D.; et al. Activating brown adipose tissue through exercise (ACTIBATE) in young adults: Rationale, design and methodology. Contemp. Clin. Trials 2015, 45, 416-425. [CrossRef]

15. Amaro-Gahete, F.J.; Jurado-Fasoli, L.; Espuch-Oliver, A.; Robles-Gonzalez, L.; Navarro-Lomas, G.; de Haro, T.; Femia, P.; Castillo, M.J.; Gutierrez, A. Exercise training as S-Klotho protein stimulator in sedentary healthy adults: Rationale, design, and methodology. Contemp. Clin. Trials Commun. 2018, 11, 10-19. [CrossRef] [PubMed]

16. Alcantara, J.M.A.; Sanchez-Delgado, G.; Martinez-Tellez, B.; Merchan-Ramirez, E.; Labayen, I.; Ruiz, J.R. Congruent validity and inter-day reliability of two breath by breath metabolic carts to measure resting metabolic rate in young adults. Nutr. Metab. Cardiovasc. Dis. 2018, 28, 929-936. [CrossRef]

17. Weir, J. New methods for calculating metabolic rate with special reference to protein metabolism. J. Physiol. 1949, 109, 1-9. [CrossRef]

18. Frayn, K. Calculation of substrate oxidation rates in vivo from gaseous exchange. J. Appl. Physiol. 1983, 55, 628-634. [CrossRef]

19. Reeves, M.M.; Davies, P.S.W.; Bauer, J.; Battistutta, D. Reducing the time period of steady state does not affect the accuracy of energy expenditure measurements by indirect calorimetry. J. Appl. Physiol. 2004, 97, 130-134. [CrossRef]

20. Matarese, L.E. Indirect calorimetry: Technical aspects. J. Am. Diet. Assoc. 1997, 97, S154-S160. [CrossRef]

21. Mullen, J.L. Indirect calorimetry in critical care. Proc. Nutr. Soc. 2005, 50, 239-244. [CrossRef] [PubMed]

22. Korth, O.; Bosy-Westphal, A.; Zschoche, P.; Glüer, C.C.; Heller, M.; Müller, M.J. Influence of methods used in body composition analysis on the prediction of resting energy expenditure. Eur. J. Clin. Nutr. 2007, 61, 582-589. [CrossRef] [PubMed]

23. Mifflin, D.; Jeor, T.; Daugherty, A.; Hill, A.; Scott, J.; Daugherty, S.; Kho, Y. A new predictive equation in healthy individuals for resting energy. Am. J. Clin. Nutr. 1990, 51, 241-247. [CrossRef] [PubMed]

24. Livingston, E.H.; Kohlstadt, I. Simplified resting metabolic rate-predicting formulas for normal-sized and obese individuals. Obes. Res. 2005, 13, 1255-1262. [CrossRef] [PubMed]

25. Müller, M.J.; Bosy-Westphal, A.; Klaus, S.; Kreymann, G.; Lührmann, P.M.; Neuhäuser-Berthold, M.; Noack, R.; Pirke, K.M.; Platte, P.; Selberg, O.; et al. World Health Organization equations have shortcomings for predicting resting energy expenditure in persons from a modern, affluent population: Generation of a new reference standard from a retrospective analysis of a German database of resting energy expenditure. Am. J. Clin. Nutr. 2004, 80, 1379-1390. [PubMed]

26. Graf, S.; Karsegard, V.L.; Viatte, V.; Maisonneuve, N.; Pichard, C.; Genton, L. Comparison of three indirect calorimetry devices and three methods of gas collection: A prospective observational study. Clin. Nutr. 2013, 32, 1067-1072. [CrossRef]

27. Forse, R.A. Comparison of Gas Exchange Measurements with a Mouthpiece, Face Mask, and Ventilated Canopy. J. Parenter. Enter. Nutr. 1993, 17, 388-391. [CrossRef]

(C) 2020 by the authors. Licensee MDPI, Basel, Switzerland. This article is an open access article distributed under the terms and conditions of the Creative Commons Attribution (CC BY) license (http://creativecommons.org/licenses/by/4.0/). 